\title{
Sampling patchy distributions: comparison of sampling designs in rocky intertidal habitats
}

\author{
A. Whitman Miller, Richard F. Ambrose* \\ Environmental Science and Engineering Program, Box 951772, University of California, Los Angeles, California \\ 90095-1772, USA
}

\begin{abstract}
Any attempt to assess species abundances must employ a sampling design that balances collection of accurate information for many species with a reasonable sampling effort. To assess the accuracy of commonly used within-site sampling designs for sessile species, we gathered cover data at 2 rocky intertidal locations in Southern California using a high-density point-contact method that maintained the spatial relationships among all points. Different sampling approaches were compared using simulated sampling. Different sampling units (single points, line transects, and quadrats) were modeled at high and low sampling efforts. Sampling units were either distributed randomly or with stratified random methods. Sampling accuracy was assessed by comparing cover and species richness estimated by the sampling simulations to the actual field data. Randomly placed single point-contacts provided the best estimates of cover but are usually not logistically feasible in the rocky intertidal, so ecologists typically use quadrats or line transects. With quadrats, some form of stratified random sampling usually gave estimates that were closer to known values than simple random placement. In nearly all stratified cases, optimum allocation of sample units, where quadrats are allocated among strata according to the amount of variability within each stratum, yielded the most accurate estimates. With 1 exception, Iine transects placed perpendicular to the elevational contours ('vertical transects') approached or exceeded the accuracy of the best stratified quadrat efforts. The estimates for rare species were consistently poor since sampling units often missed such species altogether, suggesting a systematic bias. Species richness was substantially underestimated by all sampling approaches tested, whereas these same approaches accurately estimated diversity $\left(H^{\prime}\right)$. These results illustrate the difficulty of obtaining accurate cover estimates in rocky intertidal communities.
\end{abstract}

KEY WORDS: Sampling design - Monitoring - Quadrats - Transects - Stratified random sampling . Rocky intertidal · Southern California

\section{INTRODUCTION}

Species are rarely dispersed uniformly in nature (Oosting 1956, Pielou 1977, Whittaker \& Levin 1977. Kolasa \& Pickett 1991). Instead, spatial heterogeneity is the norm, and ecological field studies and environmental monitoring programs must be designed accordingly (Green 1979, Hartnoll \& Hawkins 1980, Hurlbert 1984, Andrew \& Mapstone 1987, Eberhardt \& Thomas 1991, Underwood 1992). Although the issue of sampling patchily distributed populations cuts across all habitats and taxa, plant ecologists in particular have tried to understand the best methods for sampling

•Corresponding author. E-mail: rambrose@ucla.edu spatially variable communities (Raunkiaer 1918, Gleason 1920, Oosting 1956, Greig-Smith 1983). In recent years, issues of sampling scale and spatial heterogeneity have become central to terrestrial and marine ecologists who study community structure and function (Andrew \& Mapstone 1987, Foster 1990, Menge \& Olson 1990, Reed et al. 1993, Edmunds \& Bruno 1996).

The rocky intertidal has exceptionally high levels of spatial variability and the difficulties associated with measuring patterns of abundance in these habitats are well known (Hartnoll \& Hawkins 1980, M. N. Dethier \& L. M. Tear unpubl.). Recent work has focused on the accuracy of different methods for estimating the cover of sessile organisms within a small area (i.e., quadrat) on rocky intertidal benches, such as comparing visual 
estimates and point-contact methods (Foster et al. 1991, Meese \& Tomich 1992, Whorff \& Griffing 1992, Dethier et al. 1993, Rivas 1997). There have been few studies on how different types of sampling units (e.g., transects or quadrats) and sampling designs (e.g., random or stratified random) affect the estimates of cover over a larger region (Hartnoll \& Hawkins 1980). The development of effective sampling programs in the intertidal and other spatially heterogeneous habitats depends on how well we can expect to estimate species abundances and what sampling designs best balance sampling effort with sampling accuracy.

In this paper, we use a combination of detailed data on actual species occurrences and computer simulations to assess the accuracy of different sampling designs. Data from 2 rocky intertidal sites were repeatedly resampled, with the sampling simulation results compared to the field data to determine which design was most likely to yield estimates matching the field data. Estimates of species cover, species richness, and biological diversity were compared when sampling unit type, number, and dispersion were systematically varied. The results provide guidance about the most effective approach for sampling a heterogeneous habitat such as rocky intertidal benches, but they also highlight the difficulty of producing accurate estimates of abundance with the limited resources typically available for monitoring efforts.

Repeated sampling has been used previously to understand the efficacy of various sampling techniques in heterogeneous habitats. Before the advent of personal computers, Bauer (1943) compared the accuracy of quadrat and transect sampling techniques by manually measuring cover in artificial 'plant communities' in the laboratory (randomly placed colored cardboard disks of various abundances and sizes). Subsequent empirical field studies of a fully mapped segment of Southern California chaparral supported his prediction that lineintercept methods provided superior accuracy to quadrat methods in this plant community. Bauer had limited ability to resample and his artificial plant populations lacked the true spatial variability encountered in nature. Nonetheless, we share his goal of reducing sampling error in a spatially heterogeneous setting by understanding the consequences of different sampling approaches. Several other workers have more recently used computer simulations to evaluate different sampling issues (Wiebe 1971, Kinzie \& Snider 1978, Hellmann \& Fowler 1999). Some computer-based sampling studies relying on simulated data have focused on sampling methodology rather than sampling design (Kinzie \& Snider 1978, Dethier et al. 1993, Rivas 1997), and thus were concerned only with the distribution of organisms on the scale of an individual quadrat. Andrew \& Mapstone (1987) warn that extrapolations from computer to nature should be treated cautiously unless the simulations are constructed on a sound knowledge about the distribution and behavior of the organisms in natural conditions. For questions concerning sampling design over an entire study area, the data set should reflect the actual spatial structure of the community. We have ensured that our simulations reflect natural patterns by basing them on intensive sampling of actual rocky intertidal communities.

Using computers to simulate sampling has advantages over actual resampling. Simulated sampling offers substantial time savings and increased flexibility. Because repeating actual sampling procedures is so time-consuming, the few studies that have attempted this have been limited to only a few replicates (e.g., Foster et al. 1991, Dethier et al. 1993, Ambrose et al. 1995, Rivas 1997). The increased efficiency also allows the comparison of more alternative approaches. Simulated sampling also ensures constancy of the data set. A computer-based reference data set is fixed and therefore any differences in results are true differences. In contrast, placement or alignment errors of the measuring device in the field can introduce significant changes to the domain being sampled (Ellison 1942, Ambrose et al. 1995). In addition, the period needed for extensive intertidal ficld sampling would be protracted because sampling time is limited by appropriate tides, leading to a significant risk of actual changes in the species occurrences. In contrast, the reference data set for computer-based resampling is constant.

\section{METHODS}

Terminology. Accuracy is the closeness of a measured value to its true value; precision is the closeness of repeated measurements of the same quantity (Sokal \& Rohlf 1981). Bias refers to a systematic displacement from the true value. Unless there is a bias in measurements, precision will lead to accuracy. Thus, an accurate sampling protocol is one that produces an unbiased sample with high precision. We have focused on assessing the accuracy of different protocols.

Study sites. The 2 study sites chosen were typical of Southern California rocky bench habitats. We chose areas in the mid- to upper intertidal zone with roughly similar elevational gradients and without sharp topographical features such as high pinnacles or deep surge channels. The specific study plots were selected because they contained a variety of species (e.g., mussels, barnacles, and algae) and were typical of the surrounding bench habitat.

The White's Point study site is part of a volcanic rocky bench located on the Palos Verdes Peninsula, Los Arigeles County, California $\left(33^{\circ} 42^{\prime} 55^{\prime \prime} \mathrm{N}, 118^{\circ} 18^{\prime} 58^{\prime \prime} \mathrm{W}\right)$. 
A $10 \mathrm{~m} \times 10 \mathrm{~m}$ square study area dominated by California mussels Mytilus californianus, barnacles Chthamalus spp., including C. fissus and C. dalli, and Balanus glandula, and the red alga Endocladia muricata was measured and marked at all corners with marine epoxy. The study area is not directly exposed to breaking waves; instead, waves and surge break over the top of the promontory and flow down across the area.

A second site at Shaw's Cove, Orange County, California $\left(33^{\circ} 33^{\prime} 15^{\prime \prime} \mathrm{N}, 117^{\circ} 47^{\prime} 59^{\prime \prime} \mathrm{W}\right)$, approximately $65 \mathrm{~km}$ south of White's Point, was chosen for comparative purposes, since agreement between sites would suggest greater generalizability about sampling efficacy in Southern California rocky intertidal bench habitats. The area sampled at Shaw's Cove measured $5 \mathrm{~m} \times$ $5 \mathrm{~m}$ and was dominated by California mussels Endocladia muricata, Chthamalus spp., and non-coralline crusts. This study area has a rugose surface with cracks that maintain some standing water at low tide.

Cover measurement and reference data set production. For estimating cover of sessile invertebrates and algae, we gathered point-contact data at $10 \mathrm{~cm}$ intervals in a $1 \mathrm{~m} \times 1 \mathrm{~m}$ grid on the bench surface. Grids were aligned contiguously and covered the entire area of each study site, yielding 10000 sample points at White's Point and 2500 points at Shaw's Cove.

To locate the point contacts, a $1.2 \mathrm{~m} \times 1.2 \mathrm{~m}$ aluminum quadrat was constructed and strung with nylon-coated steel cable at $10 \mathrm{~cm}$ intervals, creating one hundred $10 \mathrm{~cm} \times 10 \mathrm{~cm}$ square cells. Point contact locations were determined with a laser pointer $(654 \mathrm{~nm}$ wavelength, $5 \mathrm{~mW}$ power output) mounted in a block that fit snugly among the wires surrounding a grid cell. When the laser block was positioned, the laser beam was centered and projected downward, normal to the grid surface. The laser cast a small red spot (approximately $2 \mathrm{~mm}$ diameter at $1 \mathrm{~m}$ height) on the bench surface, highlighting the organism to be counted. The laser block was moved sequentially from cell to cell to generate an array of data points. Besides providing unambiguous point-contact sampling without parallax problems, this quadrat design allowed the sampler to kneel beneath the frame for easier species identification. Using a laser beam instead of a sampling rod (as is frequently used with this method; Foster et al. 1991) also allowed precise sampling at distances of more than $1 \mathrm{~m}$ below the quadrat, a condition commonly encountered when sampling among surge channels, deep tide pools, steep drop-offs, and other topographical features of the intertidal. Finally, the laser system provided a simple way to align the quadrat within the study site. By placing a laser at each of the 4 quadrat corner cells and marking the contact points on the substrate below, the quadrat could be systematically moved around the site.
At White's Point and Shaw's Cove, layering of organisms was relatively infrequent. When layering was encountered, only the organism attached to the substrate was recorded. Taxonomic identification was generally done to species level, except for taxa such as encrusting algae that could not be easily identified in the field. Species contact data were gathered in sequence within each quadrat. Knowing the quadrat location and preserving the order of data collection allowed us to maintain the spatial relationship of each data point relative to all others. Species data files were imported into IDRISI (Clark University), a raster-based geographic information system, and a map describing the location of each species was generated. Sampling at White's Point was completed between December 1995 and February 1996; sampling at Shaw's Cove was completed between March and April 1996.

Simulated sampling. Cover (\%) was calculated for all species based on all point-contacts at a site. In addition to cover, we calculated species richness (the number of species) and species diversity. Diversity of biological cover (bare rock cover not included) was calculated using the Shannon-Wiener index $\left(H^{\prime}\right)$ (Shannon \& Weaver 1949).

The reference data sets were subsampled using a computer program that allowed us to locate 'virtual' transects, quadrats and single point-contacts over each study site. The sampling unit type, number, dispersion, and number of times a data set was to be resampled could be specified. In addition, sampling units could be allocated according to specified stratification criteria. The results from resampling queries were compared with actual cover values from the complete reference data sets

Six of the most abundant taxa at both sites were included in a series of sampling protocol comparisons. For each species, 2 sampling unit types were investigated, line transects and $0.5 \mathrm{~m} \times 0.5 \mathrm{~m}$ quadrats, chosen because of their common usage in rocky intertidal studies. For each simulation, multiple transects or quadrats comprised a sample. Point-contacts were recorded every $10 \mathrm{~cm}$ along a line for transects and at the same interval in an array pattern for quadrats. To simulate simple random point sampling, individual randomly located points were sampled using the White's Point data set. Sampling effort was either 300 or 1000 point-contacts ( 3 and $10 \%$ of total points) at White's Point and 150 or 250 point-contacts ( 6 and $10 \%$ of total points) at Shaw's Cove.

The sampling efforts correspond to 3 and 10 'virtual' transects (i.e., transects pulled from the computer database) at White's Point and 3 and 5 transects at Shaw's Cove. On average, transects were placed every 3.3 or $1 \mathrm{~m}$ at White's Point and every 2.5 or $1 \mathrm{~m}$ at Shaw's 
Cove. Transects were run either parallel to the site's primary slope (i.e., from the upper intertidal to the lower intertidal: 'vertical transects') or perpendicular to this slope ('horizontal transects'). For all transect protocols, transects were distributed randomly over the entire site.

The number of quadrats used for subsampling contained similar numbers of point-contacts as corresponding transect efforts $(12$ and 40 quadrats at White's Point, 6 and 10 quadrats at Shaw's Cove). Quadrats were chosen from the set of contiguous, nonoverlapping $0.5 \mathrm{~m} \times 0.5 \mathrm{~m}$ quadrats covering the entire site. Precluding quadrat overlap and not allowing quadrats to fall partly off the study site avoided artifactual sampling biases produced by edge effects. Although this model design does not incorporate every possible quadrat location, it does reflect the type of non-overlapping quadrat placement that would be used in the field. When sampled randomly, each potential quadrat location had an equal likelihood of selection.

Since organisms are not distributed evenly throughout the intertidal, the variability associated with their cover can be markedly different over a small area. A. varying spatial pattern within a study area can result in an overdil seduction in sampling precision (Andrew \& Mapstone 1987, Hayne 1987). Stratification, the subdivision of an area into more homogeneous areas with samples allocated among these subdivided areas, can be used to reduce the influence of spatial variability. The effect of stratifying quadrat location was evaluated using the White's Point data set. The study area was divided into 4 strata, with 3 main strata based on elevation and the uppermost stratum further subdivided into 2 sections based on whether or not the area fell within the splash shadow created by a large boulder.

Once stratification is imposed, a decision must still be made about how to allocate sampling units among the strata. Two methods were employed in this study. The first method simply allocated sampling units in proportion to area, regardless of any inter-stratum differences in spatial variability ('Proportional Stratified Sampling' in Andrew \& Mapstone 1987). For example, if 100 sampling units were to be allocated to 3 strata covering 20,50 , and $30 \%$ of the site, then 20 , 50 , and 30 quadrats would be allocated, respectively. The second method allocated sampling units on the basis of per-quadrat spatial variance of each species within each stratum, with more samples allocated to areas with higher variances, in a so-called 'optimum' allocation scheme (Cochran 1977; 'Stratified Sampling with Optimal Allocation' in Andrew \& Mapstone 1987). Spatial variance estimates can be cailculated using the species cover within each of the strata: $\left.v_{i j}=\sqrt{\left[\left(\operatorname{cover}_{i j}\right) \times\left(\overline{1}-\operatorname{cover}_{i j}\right)\right.}\right]$, where $v_{i j}$ denotes the spatial variance of the ith species in the $j$ th stratum. For each species, 4 variances were calculated, 1 for each stratum. These variances were then normalized by dividing each by the sum of all 4 . For example, the normalized variance for species 1 in stratum $1, V_{11}$, was calculated as $v_{11} /\left(v_{11}+v_{12}+v_{13}+v_{14}\right)$. The sample size for species 1 in stratum 1 was then calculated by multiplying the total number of sample quadrats to be allocated across the site, either 40 or 12 , by $V_{11}$. This procedure increases the precision of cover estimates by sampling more intensely in strata with more variable populations than in strata with relatively homogeneous populations. In an actual field situation, spatial variation would be estimated with the above equation and sampling units allocated accordingly. Since cover values were known for all species in each stratum at White's Point, these actual values were used to optimize quadrat allocation, which may have slightly enhanced the effects of optimum quadrat allocation over what might be realized in the field. For both allocation methods, quadrats were positioned randomly within each stratum.

Resampling. To understand the range of results typical of each sampling protocol, data sets were sampled repeatedly using a Monte Cârlo resampling method. For example, to assess how well 3 randomly placed sampling units predicted the true cover of a particular taxon, we used a computer subsampling routine to perform the following tasks: (1) For each iteration, randomly locate the 3 sample units across the study site. (2) Compare the estimated cover (or species diversity) to the true cover (or species diversity) based on the full data set. A normalized deviation from true cover $(\Delta)$ was calculated by subtracting the true cover value from the sample value (i.e., the result of each iteration) and dividing this difference by the true cover: $\Delta=$ (sample-true)/true. By normalizing cover estimates, taxa with different actual cover values can be compared to one another on identical scales. (3) Repeat this process 5000 times (or the maximum possible number of unique combinations of randomly placed sampling units). Five thousand iterations give an adequate estimate of the full range of variability in the data set (Manly 1992, unpubl. data). Thus, for each protocol tested, a frequency distribution of $5000 \Delta$ values was generated.

Since field surveys usually consist of just 1 sampling effort per location per time, analogous to a single iteration, the $\Delta$ frequency distribution can be used to indicate how close estimates from an individual sampling effort are likely to be to the actual field value. If most of the iterations are close to the true value, then a single field survey is likely to provide a good estimate of the true value. Box plots provide a visual and quantitative 
(A)

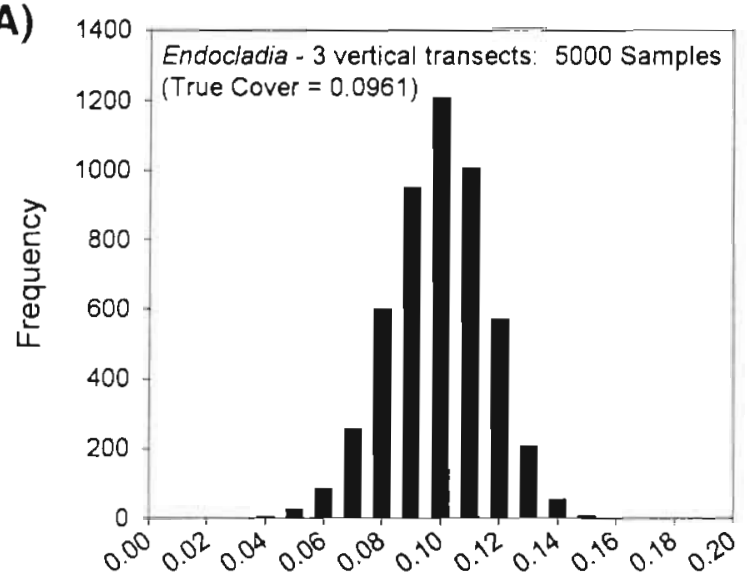

(B)

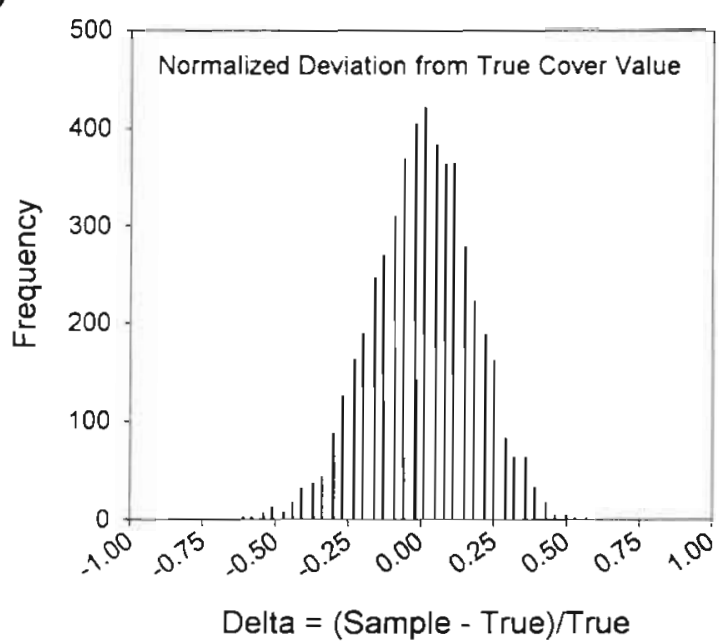

(C)

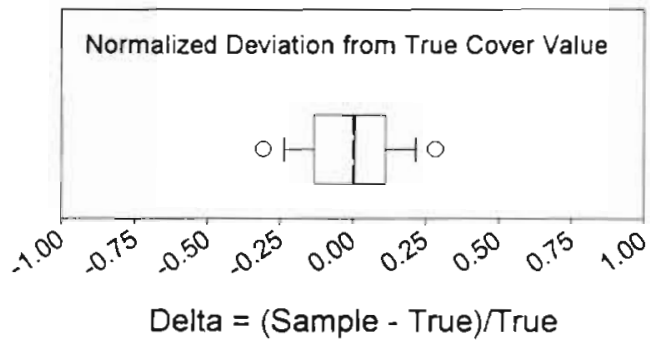

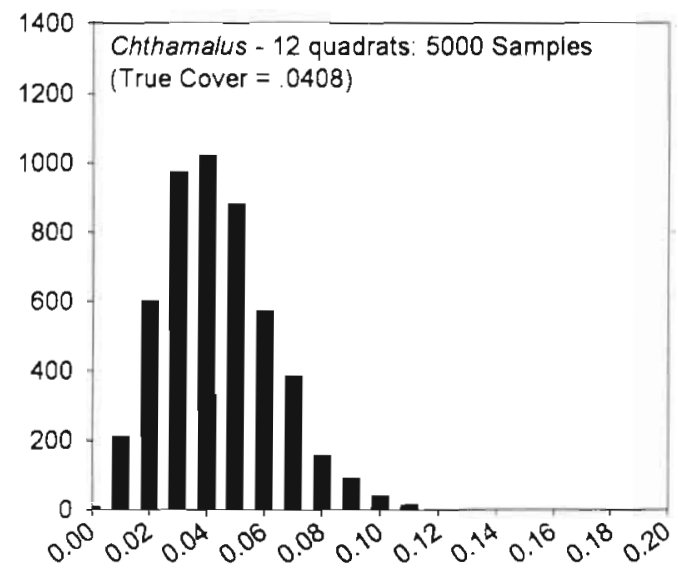

Cover Estimates
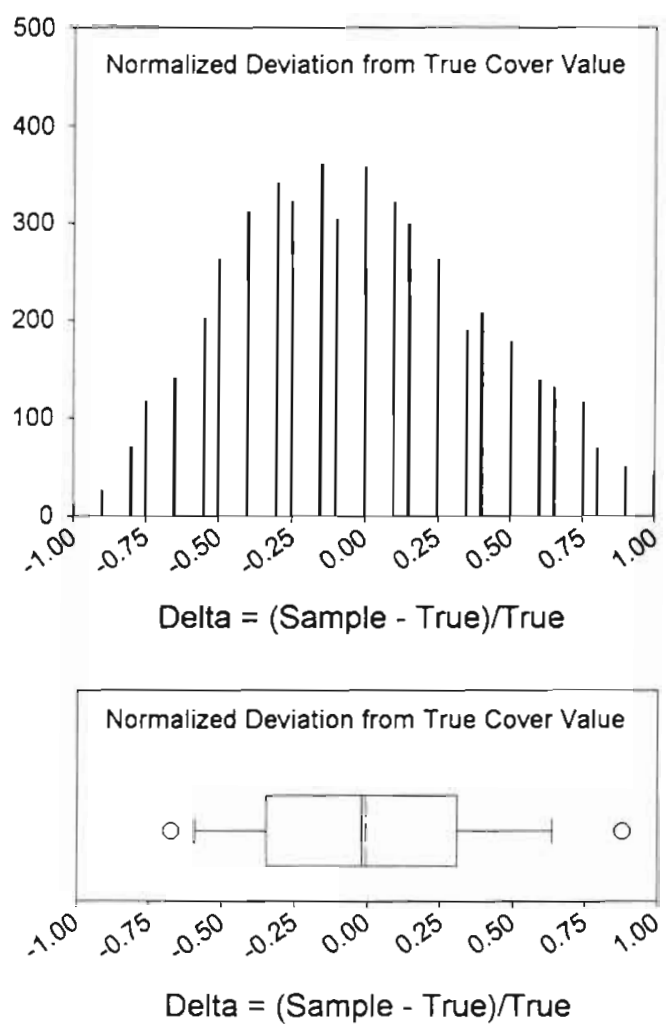

Fig. 1. Graphical summary of Monte Carlo sampling simulations and comparisons to true values. Simulations of 2 different sampling designs are illustrated with 2 species. (A) Frequency distributions of cover estimates generated by Monte Carlo simulations: units $=$ fraction of cover. (B) $\Delta$ distribution of the estimates shown in A. (C) Box plot of $\Delta$ distribution. The dashed vertical line represents mean; solid vertical line represents median; box outlines the interquartile region; whiskers (lines extending from the box) indicate 10 th and 90 th percentiles; and circles mark the 5 th and 95 th percentiles

summary of $\Delta$ distributions for easy comparison among sampling protocols.

The steps in this process are summarized in Fig. 1. Cover estimates generated by Monte Carlo simulations can be depicted as frequency distributions (Fig. 1A). The simulation results can also be presented as normalized deviations from the true cover, either as $\Delta$ distributions (Fig. 1B) or box plots (Fig. 1C). Accuracy of different sampling protocols and taxa can be compared by examining interquartile ranges in box plots. Box plots can be helpful for detecting differences in sampling precision and bias that are not readily apparent with cover fre- 
quency distributions. For example, the box plots show that most estimates of Endocladia muricata cover were closer to the true cover value than the estimates for Chthamalus spp. cover (Fig. 1C), in spite of apparently similar cover frequency distributions (Fig. 1A).

\section{RESULTS}

\section{Field data}

Thirty taxa, plus bare rock, were encountered at White's Point. Of these, 14 species accounted for over $99 \%$ of the cover there; barnacles Balanus glandula and Chthamalus spp., Endocladia muricata and $\mathrm{My}$ tilus californianus were the most common species (Table 1, Fig. 2). Fifteen taxa, in addition to rock and
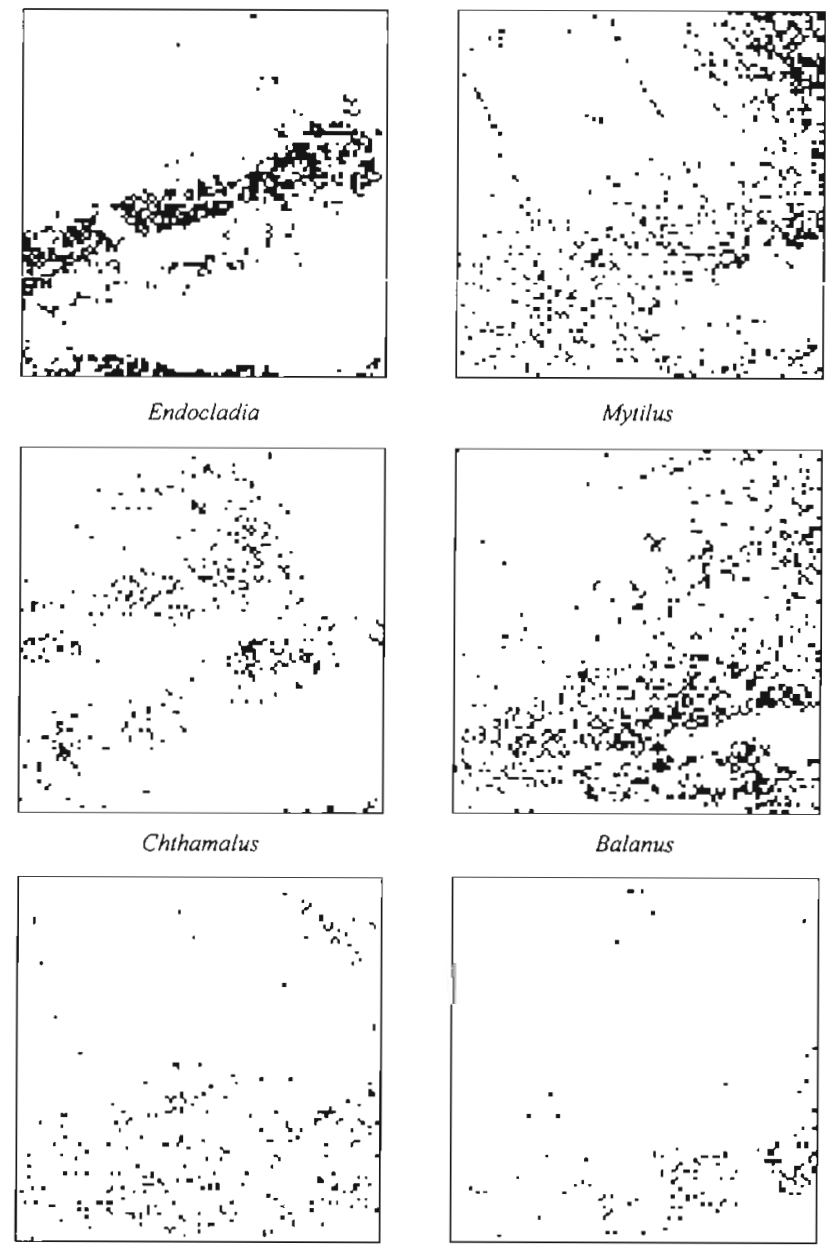

Encrusting coralline algae

Fig. 2. Coverage maps of 6 taxa at White's Point, CA. The site slopes from the top of the figure (high) to the bottom (low). Black squares represent taxon presence. The upper left-hand corner of the site, shielded from surf splash by a large boulder (just outside the study site), was dominated by bare rock tar, were encountered at Shaw's Cove. Twelve species accounted for $99 \%$ of the biological cover; in addition to the common species at White's Point, encrusting algae (coralline and Ralfsiaceae) and Pelvetia fastigiata were also common (Table 1, Fig. 3). M. californianus was much more common at Shaw's Cove. Many more rare species were encountered at White's Point. Bare rock occupied the most area at both sites.

\section{Estimates of cover from sampling simulations}

No systematic sampling bias, as indicated in box plots by sample distribution means that deviate strongly from $\Delta=0$, was found for any of the sampling protocols used to estimate cover of the common species at White's Point or Shaw's Cove (Figs. 4 \& 5).

\section{Random points}

Independent, randomly located points provide a benchmark against which to compare other sampling units and placements because individual points represent completely random sampling at each level of effort (300 and 1000 poinis). As expected, the random points provided the best estimates of cover for all species at White's Point (Fig. 4). For Anthopleura elegantissima and Endocladia muricata, there was little or no difference between random points and vertical transects (Fig. 4).

Table 1. Cover (\%) of dominant species found at 2 locations in Southern California. 'Other' species consisted of 17 species at White's Point and 5 species at Shaw's Cove

\begin{tabular}{lcc}
\hline \multirow{2}{*}{ Taxon } & \multicolumn{2}{c}{ Cover (\%) } \\
& White's Point & Shaw's Cove \\
\hline Rock & 54.95 & 34.76 \\
Balanus glandula & 10.59 & 2.20 \\
Endocladia muricata & 9.61 & 3.88 \\
Mytilus californianus & 8.58 & 25.12 \\
Chthamalus spp. & 4.08 & 7.92 \\
Anthopleura elegantissima & 2.60 & 3.60 \\
Ulva-Enteromorpha & 2.06 & 0 \\
Petrospongium rugosum & 1.75 & 0 \\
Porphyra perforata & 1.55 & 0 \\
Encrusting coralline & 1.29 & 4.40 \\
Phragmatopoma californica & 0.97 & 0.2 \\
Corallina vancouveriensis & 0.76 & 3.40 \\
Sargassum muticum & 0.27 & 0 \\
Ralfsiaceae sp. & 0.16 & 7.60 \\
Pelvetia fastigiata & 0 & 3.96 \\
Tar & 0 & 1.80 \\
Other & 0.78 & 1.16 \\
Total species & 31 & 17 \\
\end{tabular}




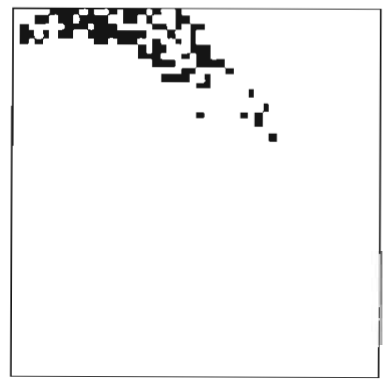

Endocladia

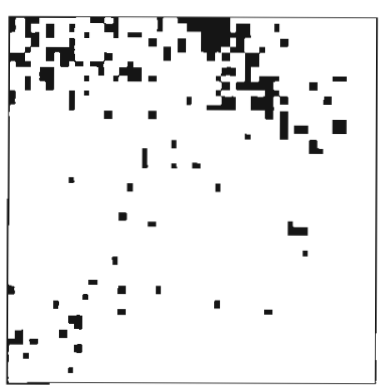

Chihamalus
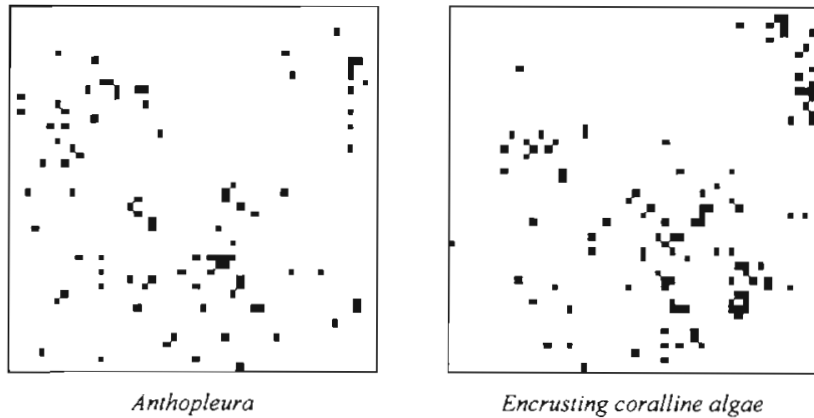

Encrusting coralline algae
Fig. 3. Coverage maps of 6 taxa at Shaw's Cove, CA. The site slopes from the top of the figure (high) to the bottom (low). Black squares represent taxon presence

Transect orientation

Vertical transects gave more accurate estimates of cover than horizontal transects for all species at White's Point except Mytilus californianus (Fig. 4). At Shaw's Cove, vertical transects gave far better sampling accuracy than horizontal transects for Endocladia muricata and Chthamalus spp., but not for the other 4 species investigated at Shaw's Cove (Fig. 5). For species occurring in low abundances at Shaw's Cove (e.g., E. muricata [3.88\%], Balanus glandula [2.04\%], and Anthopleura elegantissima [3.36\%]) sample distributions were substantially skewed from normal toward the underestimation of true cover at low sampling effort.

\section{Effects of stratification and sampling unit allocation}

Stratification at White's Point improved sampling precision over strictly random quadrat placement for all species except Chthamalus spp., which showed no improvement (Fig. 4). Quadrat allocation methods affected the results to varying degrees, depending on the species measured. Proportional quadrat allocation produced more accurate cover estimates than strict random quadrats for 5 of the 6 species at White's Point, although the improvement was minor for Mytilus californianus and Anthopleura elegantissima (Fig. 4). There was no similar improvement associated with proportional allocation for Chthamalus spp. The optimum allocation method produced more accurate cover estimates than proportional allocation for all species at White's Point except Chthamalus spp. (Fig. 4). Endocladia muricata and encrusting corallines showed the greatest improvement and Balanus glandula showed the least improvement.

\section{Transects versus quadrats}

When interquartile widths were averaged across species for each of the sampling designs, horizontal transects and random quadrats provided the poorest estimates of cover at both White's Point and Shaw's Cove (Table 2). Randomly placed parallel transects (usually vertical) gave more accurate cover estimates than random quadrats, regardless of site location (Table 2, Figs. 4 \& 5). When White's Point was stratified according to substrate elevation, more accurate cover estimates for encrusting corallines and Balanus glandula were obtained using quadrat sampling than when simple random transects were employed, but the improvement was small (Table 2).

\section{Estimates of rare species and species diversity}

Our previous analyses focused on the most common species at each study site (although even then some species had barely more than $1 \%$ cover). Because in general it is difficult to sample rare species, we also assessed how well the different sampling approaches estimated cover of a rare species and overall species diversity.

The sampling distribution for Sargassum muticum at White's Point, which had only $0.3 \%$ cover and was restricted to the small pools in the lowest section of the study site, was markedly different from the more common species (Fig. 6). In all but 1 case, the lower quartile included -1 , indicating that many of the samples did not include any $S$. muticum. However, some 
samples yielded extremely high $\Delta s$, indicating an overestimate of up to 3 -fold. Unlike the cases of skewed quartiles for more common species at Shaw's
Cove, increasing the sampling effort did not alleviate the skewness for horizontal transects or random quadrats.
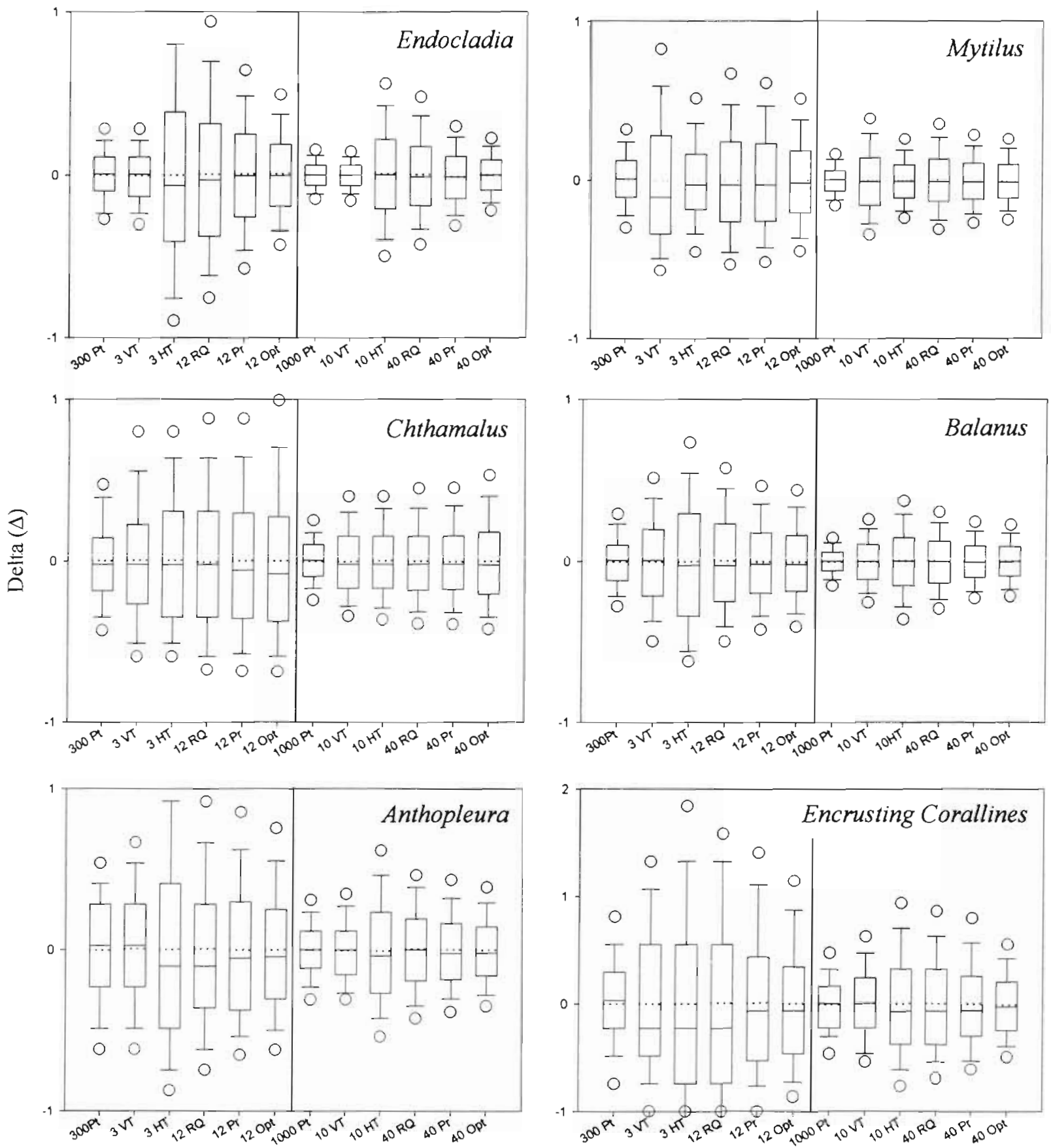

\section{Sampling Unit and Intensity}

Fig. 4. Precision and bias of different sampling approaches for White's Point, CA. Box plots show sampling distributions generated by computer subsampling of field data set. The distributions are displayed in terms of normalized cleviations of subsamples from each taxon's true percent cover, $\Delta=$ (sample-true)/true. Each distribution is based on 5000 applications of the sampling design. Sampling units: VT = vertical transects, $H T=$ horizontal transects, $R Q=$ random quadrats, $\mathrm{Pr}=$ proportionally allocated quadrats when site is stratified, Opt = optimally allocated quadrats when site is stratified. Vertical line separates low sampling effort ( $300 \mathrm{pts})$ from high effort (1000 pts) 
Species richness and species diversity were calculated for the White's Point data using random points, vertical transects, horizontal transects, and random quadrats. For species richness, precision was relatively high (i.e., the interquartile distances were narrow), but all approaches substantially underestimated the num-
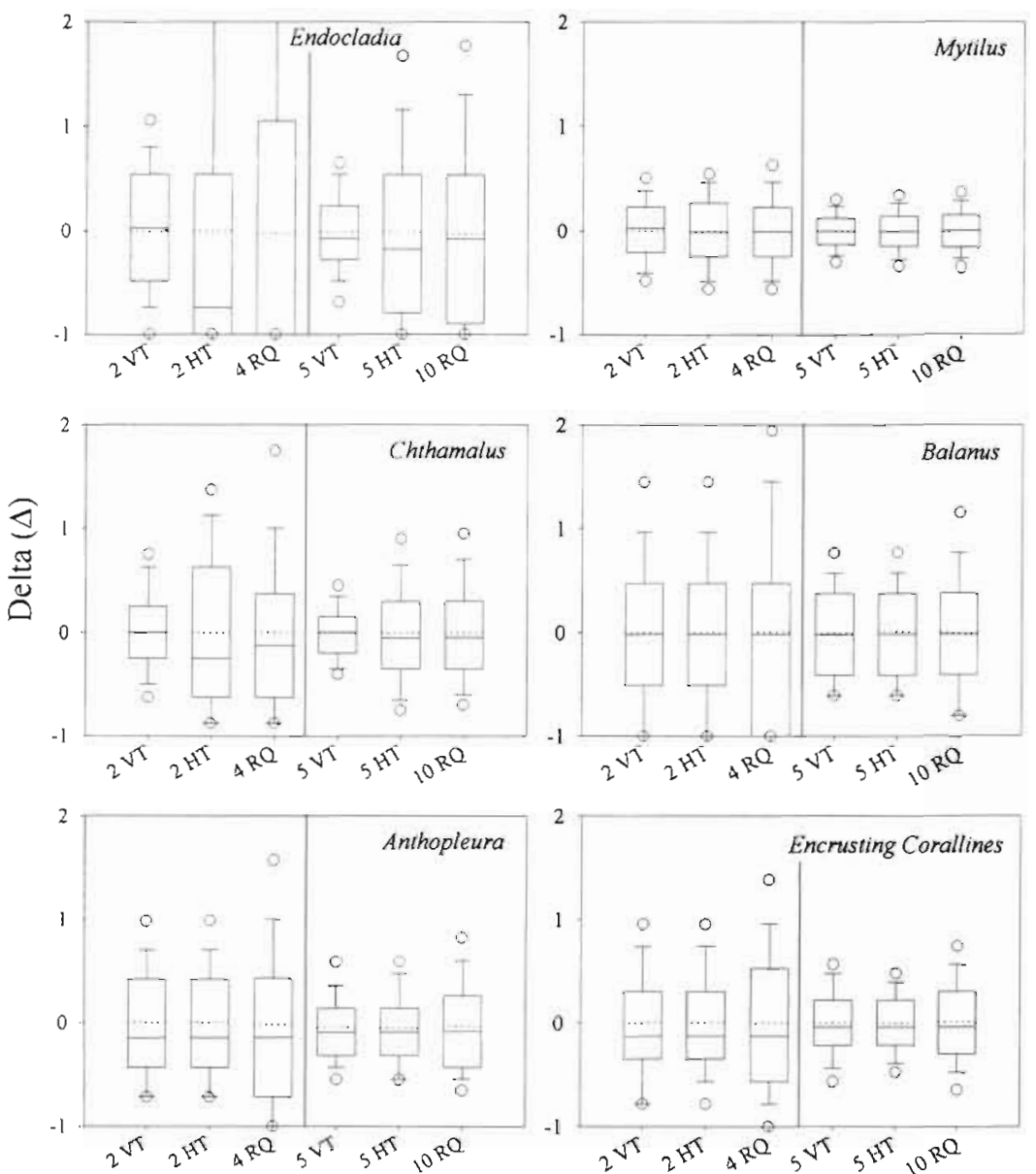

Sampling Unit and Intensity

Fig. 5. Precision and bias of different sampling approaches for Shaw's Cove, CA. Box plot characteristics and abbreviations as in Fig. 4

Table 2. Interquartile widths of $\Delta$ values among 6 taxa at White's Point and Shaw's Cove for the different sampling approaches. (a) White's Point sampling effort equaled 1000 point-contacts for all sampling techniques compared. (b) Shaw's Cove sampling effort equaled 250 point-contacts for all sampling techniques compared

\begin{tabular}{|c|c|c|c|c|c|c|c|}
\hline & $\begin{array}{l}\text { Balanus } \\
\text { glandula }\end{array}$ & $\begin{array}{c}\text { Endocladia } \\
\text { muricata }\end{array}$ & $\begin{array}{c}\text { Mytilus } \\
\text { californianus }\end{array}$ & $\begin{array}{c}\text { Chthamalus } \\
\text { spp. }\end{array}$ & $\begin{array}{l}\text { Anthopleura } \\
\text { elegantissima }\end{array}$ & $\begin{array}{c}\text { Encrusting } \\
\text { corallines }\end{array}$ & Mean \\
\hline \multicolumn{8}{|l|}{ (a) White's Point } \\
\hline Random points & 0.11 & 0.12 & 0.12 & 0.19 & 0.23 & 0.38 & 0.19 \\
\hline Vertical transects & 0.21 & 0.12 & 0.30 & 0.31 & 0.27 & 0.46 & 0.28 \\
\hline Horizontal transects & 0.29 & 0.42 & 0.20 & 0.31 & 0.50 & 0.69 & 0.40 \\
\hline Random quadrats & 0.25 & 0.36 & 0.26 & 0.32 & 0.38 & 0.69 & 0.38 \\
\hline Proportional & 0.19 & 0.25 & 0.23 & 0.32 & 0.34 & 0.55 & 0.31 \\
\hline Optimum & 0.18 & 0.18 & 0.20 & 0.38 & 0.30 & 0.44 & 0.28 \\
\hline \multicolumn{8}{|l|}{ (b) Shaw's Cove } \\
\hline Vertical transects & 0.79 & 0.51 & 0.26 & 0.34 & 0.45 & 0.43 & 0.46 \\
\hline Horizontal transects & 0.79 & 1.34 & 0.28 & 0.65 & 0.45 & 0.44 & 0.66 \\
\hline Random quadrats & 0.79 & 1.43 & 0.31 & 0.65 & 0.68 & 0.61 & 0.74 \\
\hline
\end{tabular}




\section{A. Sargassum muticum}

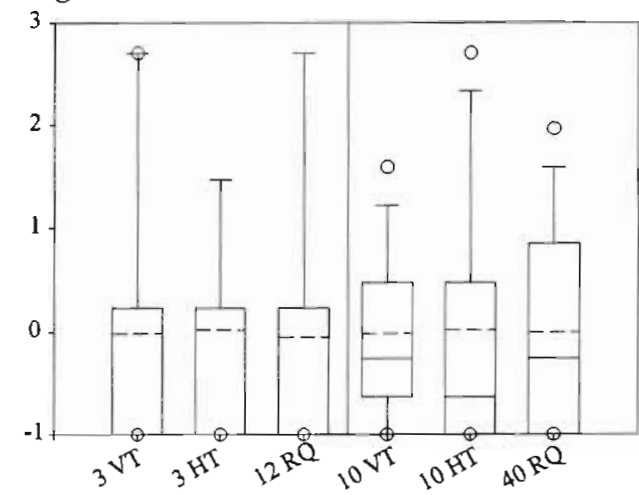

B. Species Richness

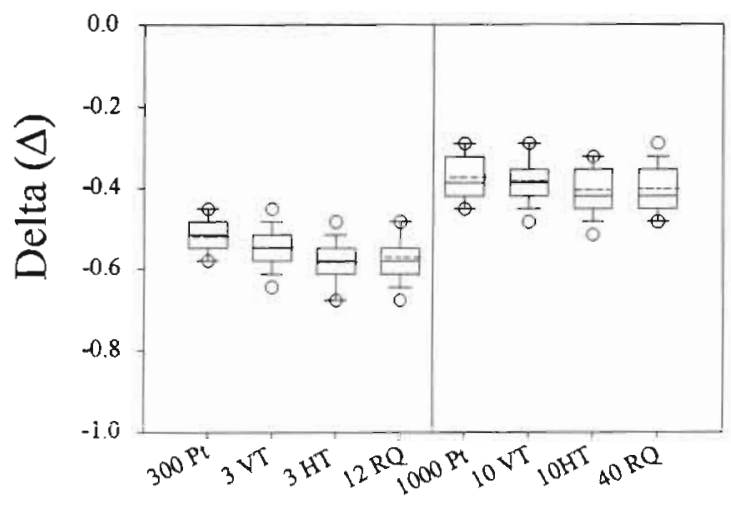

C. Shannon-Wiener Index

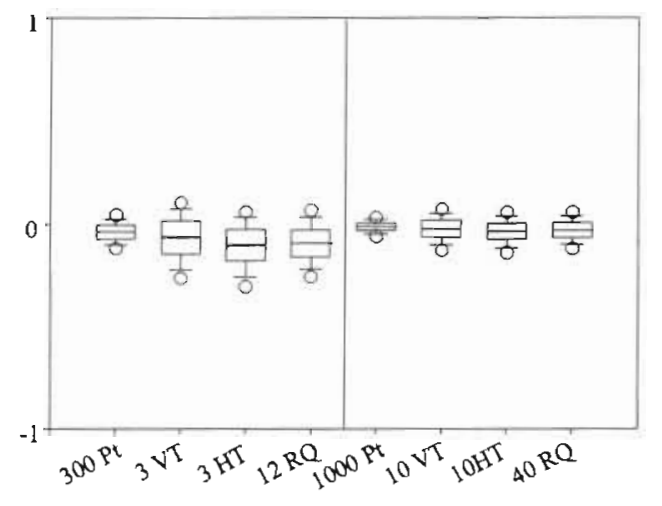

Sampling Unit and Intensity

Fig. 6. Estimates of cover and species diversity at White's Point using different sampling units and intensity. (A) Cover of Sargassum muticum, a rare species with true cover of $0.27 \%$. (B) Species richness. (C) Shannon-Wiener Index $\left(H^{\prime}\right)$.

Box plot characteristics and abbreviations as in Fig. 4

ber of species at the site, as indicated by negative biases in the sampling distributions (Fig. 6). The lower sampling effort yielded the greatest underestimate, ranging from a $50 \%$ underestimate for 300 random points to a nearly $60 \%$ underestimate for 12 random quadrats (also 300 points). Increasing the sampling effort reduced the bias, but estimated species richness still remained $40 \%$ below the actual number of species present. Only minor species richness differences were observed among various sampling designs. In all instances, none of the 5000 subsamples included the true number of species.

In comparison to estimates of species richness, estimates of $H^{\prime}$, which incorporates both species richness and evenness, were both precise and unbiased (Fig. 6). At the lower sampling effort, vertical and horizontal transects and randomly placed quadrats tended to underestimate diversity somewhat, although not as seriously as with species richness. At the higher sampling effort, all sampling designs yielded precise and unbiased (i.e., accurate) estimates of $H^{\prime}$.

\section{DISCUSSION}

Because the conclusions of field studies rest partly on how data were collected, sampling issues have received considerable attention in ecology. There has been considerable work on methods used to collect data (Levy 1933, Goodall 1952, Foster et al. 1991, Dethier et al. 1993) as well as choice of sites and sampling schedule for environmental impact assessments (e.g., Green 1979, Stewart-Oaten et al, 1986, Underwood 1994, Wiens \& Parker 1995). There has been less work on the influence of different distributions of sampling effort within a study site. As the results of this study show, within-site sampling design can have a large effect on how accurately a single sample estimates the true cover values at a site. The extent of within-site sampling error is a particular concern when a site is sampled repeatedly, either to look for the effect of an environmental impact or experimental treatment or when monitored for natural changes. If the sampling design does not provide accurate estimates, what appear to be differences from one sampling period to the next might simply reflect problems with the sampling design rather than real differences.

\section{Influence of sampling unit type and placement}

Transects and quadrats are well suited for sampling sessile or slow moving organisms and have been used extensively in terrestrial plant and intertidal and subtidal studies (Gonor \& Kemp 1978, Greig-Smith 1983). In this study, randomly located line transects consistently yielded cover estimates that were closer to the true cover than randomly placed quadrats. The greater accuracy of line transects may be related to the maximum linear dimension of the sampling unit and the spatial patterns of the species studied. All 6 species 
investigated had aggregated distributions. In this situation, sample points that are close to each other are more likely to yield similar values than points that are far apart (Hurlbert 1984, Palmer \& White 1994). Randomly placed quadrats are more likely to fall entirely within or entirely between patches than are transects. Samples with more quadrats falling by chance within patches yield an overestimate of cover, while other samples with more quadrats falling by chance between patches yield an underestimate of cover. Increasing the sampling effort improved the accuracy of the cover estimates, but for a given effort, transects provided a better estimate of cover than randomly placed quadrats.

Intertidal studies have used both vertical and horizontal transects. Given the obvious zonation of intertidal organisms, it is not surprising that directionality of transect placement influences sampling results. In species occurring in distinct bands along an elevational contour, such as Endocladia muricata at both study sites, cross-elevational transects nearly always intersect these bands, producing accurate estimates of cover. These results suggest some general guidelines about transect orientation. If a species' distribution (as visualized by 'collapsing' occurrences along 2 axes) is very clumped along 1 axis, as was the case with $E$. muricata at both sites and Anthopleura elegantissima, Balanus glandula and encrusting coralline algae at White's Point, then transects running parallel to that axis will be likely to encounter the areas of heaviest cover. In contrast, transects running perpendicular to that axis may miss these areas. In our study, vertical transects produced the best sampling accuracy in all but 1 case, and this is likely to be true often for intertidal sites given the prevalence of zonation. Thus, the elevational gradient of a site can generally be used as an effective cue for transect orientation.

When distinctly different sections exist in a study site, stratification can be used to reduce the spatial variability among individual sampling units (Cochran 1977 , Andrew \& Mapstone 1987). Intertidal researchers often use horizontal transects to improve their sampling of different zones, for example, placing 1 transect each in the high, mid- and low intertidal. Although horizontal transects may be useful for characterizing those zones, our results suggest that this approach may not provide an accurate overview of the community in the entire site.

Stratification is also used for placing quadrats. In this study, stratified random quadrats provided more accurate estimates than purely random quadrats for 5 of 6 species. Optimum quadrat allocation, which minimizes unnecessary replication in strata with low spatial variability by allocating fewer sampling units there (Cochran 1977, Andrew \& Mapstone 1987), was generally more accurate than proportional allocation. Although stratified random sampling is used frequently in ecological studies, allocation is commonly based on either equal sampling effort for each stratum or sampling effort proportional to the area of each stratum (as in our proportional allocation method); optimum allocation is rarely used.

In practice, quadrats are commonly stratified along vertical transects (Ambrose et al. 1995). This is a logistically simpler way to approach stratified random sampling; rather than having to place quadrats a random distance along 2 dimensions, distance along only 1 dimension (the transect) is varied randomly within each stratum. The cost of the simplified logistics is a greater potential for spatial autocorrelation among samples, since the quadrats are constrained to be closer to one another than would be the case for truly random stratified sampling. It seems likely that the problem of greater spatial autocorrelation will be small, but accuracy will be slightly less than with purely stratified random quadrats, which in turn will be less accurate than transects.

Understanding the effects of sample unit allocation among strata is important for ecological studies of single species. But optimizing sampling effort is far simpler in population studies than in community studies seeking to describe simultaneously the abundance of numerous taxa. Studies designed to assess the changes to community structure and composition must balance sampling effort with the overall results for a whole suite of species (Gonor \& Kemp 1978). Monitoring efforts must often use a single sampling scheme for many taxa, and in so doing have the potential for suboptimal abundance estimates for some species. Nonetheless, our results indicate that transects are likely to give a better overall description of a rocky intertidal community than random quadrats. Stratification can improve the accuracy of quadrat sampling in the intertidal, but often not to that of vertical transects for the same number of points.

Of course, the ultimate random sampling unit is the single point, and as expected randomly located points returned more accurate values than all other sampling units and placements examined in this study. The lower accuracy of random quadrats is due to the fact that quadrats constitute groupings of points, so they capture too much spatial autocorrelation. As noted above, transects spread the points over a larger area and thus are less influenced by spatial autocorrelation than quadrats, but even the points along transects are not independent. With quadrats and transects, some level of pseudoreplication, and consequently inaccurate estimate of cover, is inevitable. If single points are so clearly superior, why have so few studies utilized them? Undoubtedly because the logistics of locating 
and sampling many random individual points is too difficult and time-consuming in most systems.

\section{Accuracy}

The 'low' sampling effort used in this study is a reasonable effort for most field sampling. For example, recent baseline studies along the Southern California coast used line transects spaced every $3 \mathrm{~m}$ along a $60 \mathrm{~m}$ baseline transect (Engle et al. 1995), the same spacing used for the low-effort simulations here. At this level of effort, the precision of sample means around the true cover values (i.e., accuracy) in the present study was roughly $50 \%$. As an illustration of what this level of accuracy means, consider the case of Balanus glandula. The true value of $B$. glandula at White's Point was $10.59 \%$. If one were to sample using vertical transects every $3 \mathrm{~m}$, the first sample could easily yield an estimate of $15 \%$ (the upper quartile of the simulated sampling) and a second sample an estimate of $5 \%$ (the lower quartile). If these samples were taken on different dates, one might conclude that $B$. glandula cover had declined sharply, even though the actual cover had not changed.

As expected, higher sampling effort increased sammpling precision about the true cover value, but interquartile ranges still remained large. At White's Point, interquartile widths were 20 to $30 \%$ for most species and protocols, which means that half the time, cover estimates for most common intertidal species can be expected to be more than 10 to $15 \%$ above or below the true cover due simply to within-site sampling error. And the effort to achieve even this level of accuracy40 quadrats or 10 transects in a $10 \mathrm{~m}$ by $10 \mathrm{~m}$ area - is higher than most intertidal studies have used. Thus, the practical limit of accuracy (as indicated by interquartile ranges) for most sessile intertidal species seems to be \pm 10 to $15 \%$, based on the 6 common species used in this study.

A maximum accuracy of \pm 10 to $15 \%$ will be suitable for some purposes, but will not be acceptable if smallto-moderate differences must be detected. In particular, these results suggest that caution should be exercised when using monitoring results to indicate changes in species cover due to natural or human impacts over time. At best, monitoring programs are likely to sample at a level closer to our lower sampling effort, with an accuracy of $\pm 25 \%$ or worse. Although such a survey would provide an overview of the intertidal community at an area, it would not be sufficient to detect any but the most dramatic changes in species cover. For this reason, some monitoring programs established to detect changes in species abundances use fixed plots to reduce the high spatial variability in rocky intertidal communities, in spite of the significant limitations on inferences that can be made with fixed plots (Ambrose et al. 1995). Fixed plots do not guarantee low variability among plots (M. N. Dethier \& L. M. Tear unpubl.), but they do provide an opportunity for reducing variability and yielding good statistical power for detecting changes (Ambrose et al. 1995, R. F. Ambrose, P. T. Raimondi \& J. Spitzer unpubl. data). Given the strengths and weaknesses of the different approaches, a combination of approaches may be best for many studies.

\section{Rare species and species diversity}

The tested sampling protocols did not sample rare species well. Even species with 2 to $3 \%$ cover were frequently underestimated, particularly at the lower sampling effort. Skewed distributions suggest inadequate sample size and thus unrepresentative sampling. The central limit theorem predicts that samples from a population should be normally distributed if sampling is representative, and that deviation from normal distributions should disappear as sample sizes increase (Underwood 1997); Rice (1967) makes similar arguments for quadrat size and sampling adequacy. Tho skewness in our study was towards negative $\Delta$ s and the lower quartile often included -1.0 (indicating no contacts for that species). This type of skewness could be expected for uncommon species occurring in scattered patches; with low effort, many sets of samples would miss these patches entirely, resulting in a negative $\Delta_{\text {, }}$ while a few sets would land in the patches, resulting in a positive $\Delta$. In most cases this skewness disappeared when sample sizes were increased. However, for the least abundant species analyzed, Sargassum muticum ( $0.3 \%$ cover), even increased sampling effort did not remove the skewness for horizontal transects and random quadrats, suggesting that uncommon species need tremendous sampling effort before representative samples can be attained.

None of the sampling procedures were effective at estimating species richness for the 2 sites. This result is reminiscent of the conclusions by Dethier et al. (1993) and Rivas (1997), indicating that point-contact methods underestimate species richness compared to visual estimates. However, in this case the underestimate is not due to methodology (all comparisons were to the reference data set, and all contacts within a sampling unit were recorded without error). Rather, species richness was underestimated simply because none of the sampling efforts were sufficient to encounter many of the species actually occurring at the site. Even the higher intensity sampling underestimated species richness by about $40 \%$. These results indicate that the tra- 
ditional approaches to sampling intertidal organisms are insufficient for assessing the number of species at a site. When the goal of a monitoring effort is biodiversity assessment, accurate estimates of species richness may require supplemental sampling specifically for that purpose.

In contrast to the poor estimates of species richness, all sampling approaches yielded quite accurate estimates of species diversity. $H^{\prime}$ incorporates both richness and evenness, but is sensitive to the inclusion of rare species (i.e., species richness) compared to other diversity indices (Magurran 1988). Since we know that species richness was underestimated by $\geq 40 \%$ in the same samples that estimated diversity to within $10 \%$ of the true value, increased evenness must have compensated for the lower species richness. It is not clear, however, whether this result will generally be true, or whether the near-perfect balancing of lower richness and increased evenness in this analysis was simply a coincidence. The fact that diversity was estimated more accurately than species richness suggests it might be a useful monitoring parameter, but further work should be done to confirm this conclusion.

\section{Conclusions}

Simple decisions about sampling approach (choice of sampling unit and allocation of sampling effort over space) can influence abundance estimates significantly. The accuracy of different sampling approaches compared in this study typically varied 2 - or 3 -fold. In the rocky intertidal communities studied here, vertical line transects generally provided the greatest accuracy. Besides being more accurate, line transects frequently require less field time for sampling than quadrat schemes (pers. obs.). Since the reference database used for our analyses was based on actual field occurrences and there was consistency between the two study sites, it seems likely that these results are representative of a wider variety of rocky intertidal and perhaps other habitats.

Even the best practical sampling design is likely to have limited accuracy in habitats with as much spatial variability as the rocky intertidal. Half the time, a reasonable sampling effort using the best sampling unit and placement would likely yield estimates of the mean that are more than $25 \%$ higher or lower than the true mean. Tripling the sampling effort may improve the precision from \pm 25 to $\pm 10-15 \%$ for common species, but this probably represents a practical limit. Even at this higher intensity, the common sampling approaches cannot reliably estimate the cover of rare species or the species richness of a site. For general surveys, a rough cover estimate of common species may be sufficient for an overview of a rocky intertidal community. However, for many purposes, such as environmental monitoring, impact assessment, and biodiversity surveys, this level of accuracy is likely to be insufficient, and alternative approaches (e.g. fixed plots or visual scans) will be required.

Acknowledgements. We thank K. Klug for invaluable computer programming, S. Anderson, S. Daily, Mariel Hagan, Melissa Hagan, T. Ludwig, A. Miller, H. Miller, N. Mode, and P. Pless for field assistance, F. Miller and D. Miller for design and construction of sampling equipment, W. Cumberland, L. D. Duke, R. Vance, N. Mode, and K. Lafferty for comments during this project, and M. Dethier, S. Murray, and P. Raimondi for comments on an earlier version of the manuscript. This research was funded by the California Coastal Commission through the Southern California Educational Initiative at the University of California, Santa Barbara; we thank Zach Hymanson and Susan Hansch of the Commission for their assistance and support.

\section{LITERATURE CITED}

Ambrose RF, Engle JM, Raimondi PT, Wilson M Altstatt J, (1995) Rocky intertidal and subtidal resources: Santa Barbara County mainland. Report to the Minerals Management Service, Pacific OCS Region. OCS Study MMS 950067, Minerals Management Service, Camarillo, CA

Andrew NL, Mapstone BD (1987) Sampling and the description of spatial pattern in marine ecology. Oceanogr Mar Biol Annu Rev 25:39-90

Bauer HL (1943) The statistical analysis of chaparral and other plant communities by means of transect samples. Ecology 24:45-60

Cochran WG (1977) Sampling techniques. John Wiley \& Sons, Inc, New York

Dethier MN, Graham ES, Cohen S, Tear LM (1993) Visual versus random-point percent cover estimations: 'objective' is not always better. Mar Ecol Prog Ser 96:93-100

Eberhardt LL, Thomas JM (1991) Designing environmental field studies. Ecol Monogr 61:53-73

Edmunds PJ, Bruno JF (1996) The importance of sampling scale in ecology: kilometer-wide variation in coral reef communities. Mar Ecol Prog Ser 143:165-171

Ellison L (1942) A comparison of methods of quadratting short-grass vegetation. J Agric Res 64:595-614

Engle JM, Lafferty KD, Dugan JE, Martin DL, Mode $N$, Ambrose RF, Raimondi PT (1995) Second year study plan for inventory of coastal ecological resources of the northern Channel Islands and Ventura/Los Angeles Counties Report to the California Coastal Commission, California Coastal Commission, San Francisco, CA

Foster MS (1990) Organization of macroalgal assemblages in the northeast Pacific: the assumptions of homogeneity and the illusion of generality. Hydrobiologia 192:21-33

Foster MS, Harrold C, Hardin DD (1991) Point vs photo quadrat estimates of the cover of sessile marine organisms. J Exp Mar Biol Ecol 146:193-203

Gleason HA (1920) Some applications of the quadrat method. Bull Torrey Bot Club 47:21-33

Gonor JJ, Kemp PF (1978) Procedures for quantitative ecolog- 
ical assessments in intertidal environments. US Environmental Protection Agency Report EPA-600/3-78-087, U.S. Environmental Protection Agency, Corvallis, OR

Goodall DW (1952) Some considerations in the use of point quadrats for the analysis of vegetation. Aust J Sci Res Ser B 5:1-141

Green RH (1979) Sampling design and statistical methods for environmental biologists. John Wiley \& Sons, New York

Greig-Smith P (1983) Quantitative plant ecology. University of California Press, Berkeley, CA

Hartnoll RG, Hawkins SJ (1980) Monitoring rocky-shore communities: a critical look at spatial and temporal variation. Helgol Meeresunters 33:484-494

Hayne DW (1987) Development of environmental data bases and inventories. In: Draggan S, Cohrssen JJ, Morrison RE (eds) Environmental monitoring, assessment, and management (the agenda for long-term research and development). Praeger Publishers, New York, p 27

Hellmann JJ, Fowler GW (1999) Bias, precision and accuracy of four measures of species richness. Ecol Appl 9:824-834

Hurlbert ST (1984) Pseudoreplication and the design of ecological field experiments. Ecol Monogr 54:187-211

Kinzie RA, Snider RH (1978) A simulation study of coral reef survey methods. In: Stoddart DR, Johannes RG (eds) CoraI reefs: research methods. Monographs in oceanographic methodology No. 5. UNESCO, Paris, p 231-250

Kolasa J, Pickett STA (eds) (1991) Ecological heterogeneity. Springer-Verlag, New York

Levy BE (1933) The point method of pasture analysis. NZ J Agric 46:267-279

Magurran AE (1988) Ecological diversity and its measurement. Princeton University Press, Princeton, NJ

Manly BFJ (1992) Randomization and Monte Carlo methods in biology. Chapman \& Hall, London

Meese RJ, Tomich PA (1992) Dots on rocks: a comparison of percent cover estimation methods. J Exp Mar Biol Ecol 165:59-73

Menge BA, Olson AM (1990) Role of scale and environmental factors in regulation of community structure. Trends Ecol Evol 5:52-57

Oosting HJ (1956) The study of plant communities (an introduction to plant ecology), 2nd edn. WH Freeman and Co, San Francisco, CA

Editorial responsibility: Lisa Levin (Contributing Editor), La Jolla, California, USA.
Palmer MW, White PS (1994) Scale dependence and the species-area relationship. Am Nat 144:717-740

Pielou EC (1977) Mathematical ecology. John Wiley \& Sons, New York

Raunkiaer C (1918) Recherches statistiques sur les formations vegetales. Biol Medd K Dan Vidensk Selsk 1:1-80

Reed RA, Peet RK, Palmer MW, White PS (1993) Scale dependence of vegetation-environment correlations: a case study of a North Carolina piedmont woodland. J Veg Sci 4: 329-340

Rice EL (1967) A statistical method for determining quadrat size and adequacy of sampling. Ecology 48:1047-1049

Rivas OO (1997) Laboratory evaluation of a video method for sampling rocky intertidal populations. MSc thesis, California State University, Fullerton, CA

Shannon CE, Weaver W (1949) The mathematical theory of communication. University of Wlinois Press, Urbana, IL

Sokal RR, Rohlf FJ (1981) Biometry: the principles and practice of statistics in biological researc. WH Freeman and Co, San Francisco, CA

Stewart-Oaten A, Murdoch WW, Parker KR (1986) Environmental impact assessment: 'Pseudoreplication' in time? Ecology 67:929-940

Underwood A.J (1992) Beyond BACI: the detection of environmental impacts on populations in the real, but variable world. J Exp Mar Biol Ecol 161:145-178

Underwood AJ (1994) On beyond BACI: sampling designs that might reliably detect environmental disturbances. Ecol Appl 4:3-15

Underwood AJ (1997) Experiments in ecology: their logical design and interpretation using analysis of variance. Cambridge University Press, Cambridge

Whittaker RH, Levin SA (1977) The role of mosaic phenomena in natural communities. Theor Pop Biol 12:117-139

Whorff JS, Griffing L (1992) A video recording and analysis system used to sample intertidal communities. J Exp Mar Biol Ecol 160:1-12

Wiebe PH (1971) A computer model study of zooplankton patchiness and its effects on sampling error. Limnol Oceanogr 16:29-38

Wiens JA, Parker KR (1995) Analyzing the effects of accidental environmental impacts: approaches and assumptions. Ecol Appl 5:1069-1083

Submitted: May 13, 1999; Accepted: October 11, 1999

Proofs received from author(s): March 27, 2000 